\title{
Integrated CPU and Network-I/O QoS Management in an Endsystem
}

\author{
K. Lakshman, Raj Yavatkar
}

Intel Architecture Labs

2111 N.E 25th Avenue, Hillsboro, OR 97124.

lakshman@ibeam.intel.com,yavatkar@ibeam.intel.com

Raphael Finkel

University of Kentucky, Department of Computer Science

773 Anderson Hall, Lexington KY 40506, raphael@cs.uky.edu

\begin{abstract}
Realtime multimedia applications such as conferencing, broadcast video, and distributed virtual reality demand predictable QoS from both endsystem and network resources. We show how to provide predictable QoS for multimedia applications in an environment where applications do not know the exact resource requirements in advance and where both resource requirements and resource availability change at runtime. We propose a resource management architecture in which applications and the OS cooperate to dynamically adapt to variations in the resource requirements and availability. We have implemented the resulting OS architecture, called AQUA (Adaptive Quality of service Architecture), in the Solaris OS, and have used it to manage CPU and network-I/O resources in an integrated fashion.
\end{abstract}

Keywords

Keywords: endsystem, CPU, I/O, Solaris, adaptive QoS

\section{INTRODUCTION}

The recent trend in increasing power and versatility of a PC has led to a new class of performance-conscious, realtime multimedia applications such as audio/video conferencing, broadcast quality presentations, and distributed virtual reality. Such applications demand predictable quality of service (QoS) from endsystęm and network resources.

In order to provide predictable $\mathrm{QoS}$ to such applications in a single-user environment, we propose a cooperative model of resource management in 
which both applications and OS dynamically cooperate to adaptively share endsystem resources.

Our approach is motivated by the following observations about multimedia applications and their endsystem environment.

Resource requirements vary dynamically: The resource requirements of variable bit rate applications such as compressed audio/video players or servers cannot be fully determined in advance and tend to vary dramatically over their life time (Yavatkar \& Lakshman 1995). Although a video player's desired rate of execution can be specified in advance, the amount of CPU time or bandwidth needed over a time interval may vary dramatically. Such applications must be able to dynamically renegotiate their $Q o S$ requirements.

Resource availability varies dynamically: On an endsystem, resource availability dynamically depends on the current mix of applications. For instance, interactive collaboration applications and distributed games combine individual multimedia applications that concurrently generate or play back multiple audio and video streams. As the resource requirements of individual streams change and as applications are started or terminated, the amount of available resources for other applications changes. Applications must be prepared to dynamically adapt to changes in resource availability.

Multimedia applications use multiple resources: A typical multimedia application needs predictable service from more than one resource to achieve its desired QoS. A video player must be able to execute periodically and obtain the necessary amount of CPU time, memory, bus bandwidth, and network bandwidth to successfully receive a video stream, uncompress it, and display it. Use of one resource typically leads to an indirect use of another resource. For example, predictable network-I/O protocol processing requires predictable access to $\mathrm{CPU}$. The OS must include integrated resource management mechanisms that can handle such inter-dependencies.

Multimedia applications are amenable to graceful adaptation: Unlike traditional hard realtime applications, video or audio applications can generally adapt to fit within available resources by changing media characteristics. For example, a video application can adapt by changing its resource requirements across three dimensions: the frame rate (temporal resolution), the frame size (spatial resolution), and the pixel depth (visual resolution).

The relative priority of applications changes dynamically: In a single-user environment, the relative importance of one application over another varies dynamically. For example, when the user switches from watching a news broadcast to a compilation task, the news application may execute at a lower priority. Applications should be able to temporarily reduce their resource requirements and switch back to full activity when needed.

Our thesis is that the resources on an endsystem should be allocated and managed adaptively in an integrated manner to accommodate the variations in both the QoS requirements and the resource availability. We propose an 
OS architecture called AQUA (Adaptive Quality of service Architecture) for managing resources to provide predictable QoS.

AQUA uses a cooperative model of resource management; applications (resource consumers) and the OS (the resource provider) cooperate to manage resources in the following way.

When an application starts, it provides a partial QoS specification (for example, a video player wishes to execute 30 times a second, but might not specify its compute and bandwidth requirements). The resource provider allocates a resource based on its estimate of the available capacity.

As the application runs, the resource provider and consumer cooperate to estimate the application's resource requirements and the QoS it receives. As resource requirements vary (a scene change alters the CPU requirements of a video player) or resource availability changes (other applications start or stop), resulting changes to measured QoS are detected, and both the resource consumer and provider renegotiate and adapt to ensure predictable service within the constraints of available resources.

We have implemented AQUA in the Solaris 2.4 operating system and evaluated it in an environment consisting of Sun Sparc workstations and a set of ATM switches. This paper describes how AQUA manages CPU and networkI/O resources. Section 2 provides an overview of resource management. Section 3 describes management of CPU. Section 4 describes integrated resource management for providing network-I/O QoS. Section 5 describes related work. Finally, Section 6 summarizes our contributions.

\section{THE AQUA FRAMEWORK}

AQUA's model of resource management assigns an active role to the applications in allocating and managing resources. To avoid burdening application programmers with the details of QoS negotiation and adaptation and to encourage code reusability, AQUA includes abstract interfaces and libraries that hide the details of QoS measurement and negotiation. These interfaces and libraries include an application-level QoS manager, a QoS negotiation library, and a usage-estimation library.

A resource specific QoS manager is linked with the application code (Figure 1). It exports a QoS-specification interface to the application programmer and hides the details of negotiation with the resource providers. When an application provides a partial QoS specification, the QoS manager handles the details of requesting resource allocation from the resource controllers. As the application runs, the QoS manager estimates the resource usage and monitors the QoS achieved based on the feedback from the resource provider. When the measured QoS does not meet the desired QoS (adequate resources are not available) or additional resources become available, the QoS manager initiates adaptation by invoking an adaptation function supplied by the application.

Two utility libraries handle common tasks for the QoS manager. The nego- 


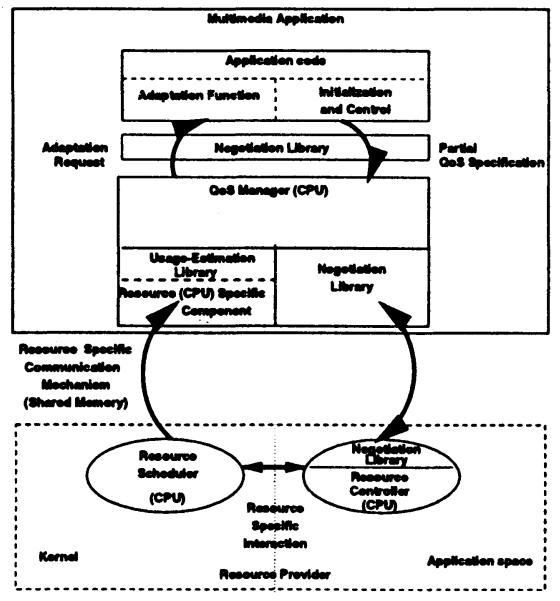

Figure 1 AQUA components: an application-level QOS manager, a resource controller for each resource, and libraries for communication between them.

tiation library hides the details of QoS negotiation with a resource provider. The usage-estimation library helps the QoS manager estimate resource usage and QoS delivery and includes a resource specific component. It interacts with the resource providers and gathers resource-usage and QoS-delivery statistics.

\section{THE CPU RESOURCE}

We will use the CPU resource as an example to describe the details of the AQUA framework. We implemented the RAP (Rate-based Adjustable Priority) CPU scheduling policy (Yavatkar \& Lakshman 1995), which extends rate-monotonic scheduling to account for unknown and varying compute times and global adaptation across many applications. As shown in Figure 1, CPU resource management includes CPU specific components: a CPU QoS manager, a CPU-specific usage-estimation library component, the CPU resource controller (RAP controller), and the CPU scheduler (RAP scheduler in the kernel). The RAP controller implements admission control and participates in dynamic QoS negotiation with the CPU QoS manager.

\subsection{A Simple Video Server}

Figure 4 shows code for a simple video server that wishes to execute thirty times per second to capture, compress, and send video frames. For simplicity, we ignore the details of network $1 / 0$, which we discuss in a later section. Figures 1-3 show the various components of AQUA used by this application. The video server executes the following sequence.

It first invokes the AQUA initialization functions to obtain a handle to the CPU resource and starts a CPU QoS manager. It then supplies a partial 


\begin{tabular}{|c|c|}
\hline Turn resource name into unique id & id_t GetResByHame (name) \\
\hline $\begin{array}{l}\text { Request allocation of a resource } \\
\text { using the given qos parameter }\end{array}$ & $\begin{array}{l}\text { error_t GetRes (resid,*qos, } \\
\text { qos_len) }\end{array}$ \\
\hline Release the use of a resource & void RelRes (resid) \\
\hline $\begin{array}{l}\text { Update resource reservation } \\
\text { using the given qos }\end{array}$ & $\begin{array}{l}\text { error_t UpdateqoS (resid, } \\
\quad * q 0 s, \text { qos_len) }\end{array}$ \\
\hline $\begin{array}{l}\text { Request notification when } \\
\text { a resource becomes available. } \\
\text { The qos parameter contains } \\
\text { the amount of resource needed }\end{array}$ & $\begin{array}{l}\text { error_t NotifyqoS(resid, } \\
\quad * q 0 s, q 0 s_{-1} \text { en) }\end{array}$ \\
\hline $\begin{array}{l}\text { Register a function for } \\
\text { handling adaptation requests }\end{array}$ & $\begin{array}{l}\text { void CBreg_Adapt (resid, } \\
\text { (*func) }(), \text { arg) }\end{array}$ \\
\hline
\end{tabular}

Figure 2 Generic resource-negotiation calls provided by the negotiation library for resource consumers. The exact contents of the qos specification is resource specific, examples are given in (Lakshman 1997)

\begin{tabular}{|c|c|}
\hline $\begin{array}{l}\text { Register resource } \\
\text { name }\end{array}$ & id_t RegName (name); \\
\hline $\begin{array}{l}\text { Register callback } \\
\text { handler functions } \\
\text { for aqua primitives }\end{array}$ & $\begin{aligned} & \text { void } \text { CBbreg_Hndlrs (id, } \\
& \text { (*GetRes_func) }(), \\
& \text { (*RelRes_func) }(), \\
& \text { (*UpdateqoS_func) }(), \\
&\text { (*NotifyqoS_func) }()) ;\end{aligned}$ \\
\hline $\begin{array}{l}\text { Request a consumer } \\
\text { specified by } \\
\text { id to adapt }\end{array}$ & $\begin{array}{l}\text { void AdaptReq(id, } \\
\text { *qos, } \\
\text { q0s_len }\end{array}$ \\
\hline $\begin{array}{l}\text { Wait for a response } \\
\text { to an adaptation } \\
\text { request }\end{array}$ & $\begin{array}{l}\text { id_t AdaptReq_Resp (*qos, } \\
\text { q0s_len }\end{array}$ \\
\hline
\end{tabular}

\begin{tabular}{|c|c|}
\hline $\begin{array}{l}\text { Register } \\
\text { callback } \\
\text { function to } \\
\text { monitor } \\
\text { QoS }\end{array}$ & $\begin{array}{l}\text { CBreg_Monitor(resid, } \\
\text { (*func) (), } \\
\text { caddr_t arg, } \\
\text { time_t time) }\end{array}$ \\
\hline $\begin{array}{l}\text { Get } \\
\text { resource } \\
\text { usage }\end{array}$ & $\begin{array}{l}\text { GetUsage (resid, } \\
\text { *qos, } \\
\text { qos_len) }\end{array}$ \\
\hline $\begin{array}{l}\text { Register } \\
\text { filter } \\
\text { callback } \\
\text { function }\end{array}$ & $\begin{array}{c}\text { CBreg_Filter (resid, } \\
\text { ( } * \text { func) }(), \\
\text { caddr_t arg })\end{array}$ \\
\hline
\end{tabular}

Figure 3 A: Calls provided by the negotiation library to allow resource controllers and QoS managers to communicate with resource consumers and application code. B: Functions provided by the usage-estimation library

QoS specification containing the desired rate of execution, the averaging interval for measuring the rate, and an acceptable rate jitter. It cannot specify the compute time needed. It registers a function for adaptation (CPU_Adapt), which is specific to an application and includes application-specific semantics for adaptation. For instance, the video server may first adapt by reducing its frame rate followed by a change in its frame size if necessary. It repeatedly executes its main body, which is a loop that grabs a frame, packetizes it, and sends it. It indicates the end of each iteration by yielding.

As the video server executes, various components interact as follows.

1. The video server provides a partial QoS specification, and the QoS manager fills in any unspecified QoS parameters that it can determine beforehand and invokes the RAP controller for initial resource allocation (GetRes).

2. The RAP controller scans RAP kernel queues to determine the available 


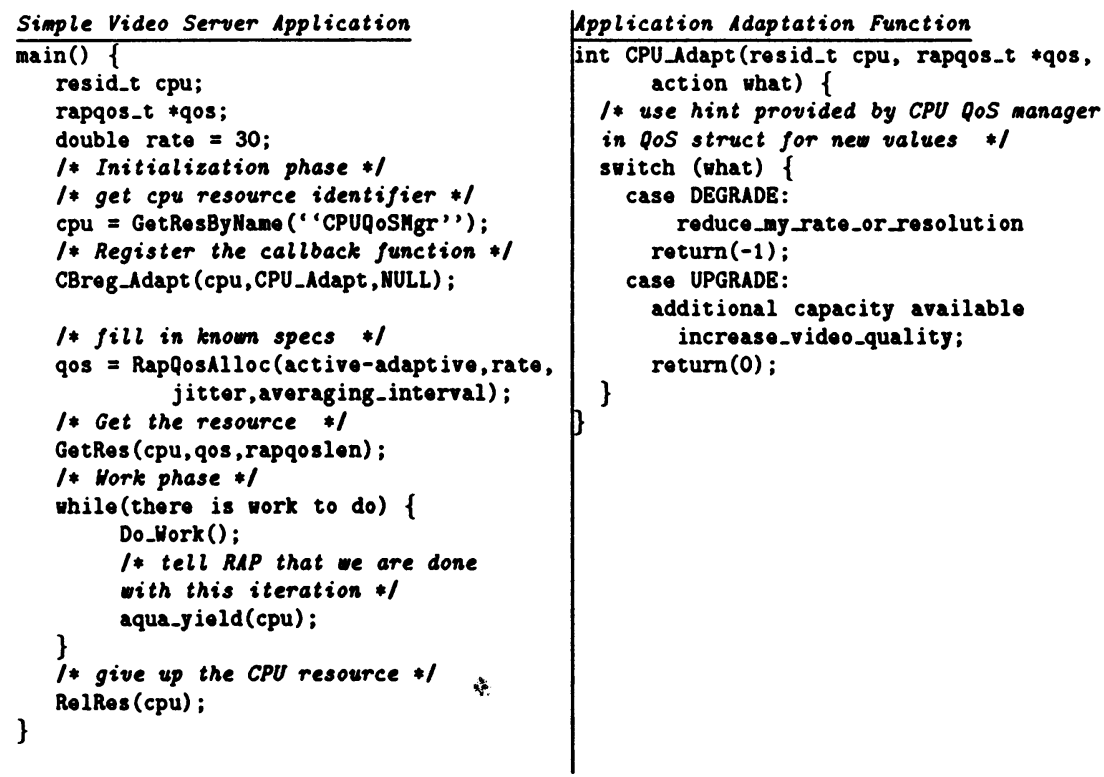

Figure 4 A AQUA video server. If its resource requirements exceed available CPU resource, it adapts by reducing either its rate or its spatial resolution. When more resources become available, it adapts by improving video quality.

CPU capacity (compute time slack). It allocates all available slack (but not more than 10 ms on a sparc 20) to the new thread. The compute time actually used will likely stabilize to a lower value in a few averaging intervals.

3. The QoS manager monitors the resource usage and QoS received every averaging interval via the GetUsage call in the usage-estimation library. This library keeps track of the compute time needed per period by collecting samples over an averaging interval. When the video server yields at the end of each execution period, the usage estimation library accesses kernel data structures to learn the amount of compute time used in that iteration. It then calls a low-pass filter in the QoS manager to compute a long-term average. It also records the observed rate of execution.

4. The QoS manager uses the UpdateQoS call to update the RAP controller's initial estimate of the compute time by giving it the filtered compute-time requirement. The RAP controller now has a reliable estimate of the compute time used; so it can add excess unused capacity to the slack estimate.

5. If the QoS manager observes a change in CPU usage, it updates its reservation with the RAP controller (UpdateQoS).

6. The QoS manager compares the QoS delivered with the requested QoS. If the delivered level of QoS is less than that requested, the QoS manager requests an increased allocation of compute time from the RAP controller. If the RAP controller is unable to meet the new requirements, the QoS manager asks the video player to change its behavior by invoking the video player's adaptation function (AdaptReq).

7. When several applications compete for CPU and adequate aggregate ca- 
pacity is not available, the RAP controller may resort to global adaptation either by reducing the resource allocation of each admitted application (transparent adaptation) or by explicitly requesting that each application reduce its resource requirements (active adaptation) (AdaptReq).

\section{INTEGRATED CPU AND NETWORK-I/O MANAGEMENT}

Actual applications typically need more than just the CPU resource. For example, a video server needs predictable QoS from both CPU and network-I/O resources. Network I/O indirectly requires other resources, such as CPU for protocol processing, I/O-bus bandwidth for transferring data between memory and network-I/O devices, and the network interface for transmitting data.

The AQUA framework integrates resource management for CPU and network$\mathrm{I} / \mathrm{O}$ resources. Integrated resource management is achieved by using a composite QoS manager that uses the services of both the network-I/O QoS manager and the CPU QoS manager. The application specifies its QoS requirements to the composite QoS manager. Each QoS manager is responsible for determining the application's requirements for its resource. The QoS managers interact with their corresponding resource controllers to negotiate the QoS.

The network-I/O provider consists of a network-I/O controller, responsible for admission control and global adaptation, and the network-interface driver, which controls link scheduling.

In the following, we describe our implementation of an integrated resource manager that provides predictable QoS for network data transfer by managing both CPU and network I/O subsystems in the Solaris OS. Although data transfer uses bus bandwidth, we have not yet implemented a bus-bandwidth management component of the integrated resource manager.

To ensure that the interrupt and protocol processing is done in a timely fashion in the OS, we addressed three problems that arise in the Solaris OS: (1) When many processes concurrently transfer data streams across the protocol stack, each data transfer affects the predictibility of other data transfers (the inter-stream interference problem). (2) When a process requests an I/O operation such as reading a block of data, the $\mathrm{I} / \mathrm{O}$ is performed in the background using asynchronous, interrupt-driven $\mathrm{I} / \mathrm{O}$. When an $\mathrm{I} / \mathrm{O}$ interrupt occurs, the currently executing process is interrupted and delayed, which makes its execution unpredictable (the $I / O$ interference problem). (3) To allocate and account for the CPU time used for protocol processing, the QoS manager must be able to estimate the CPU requirements of protocol processing.

Figure 5 illustrates the impact of these problems. Process 1 is a realtime application that sends data over the ATM network. It competes with process 2 , a nonrealtime application that sends data over a $100 \mathrm{Mbps}$ ethernet. After process 2 starts at time 30 , process 1 is unable to receive the QoS it requires (send-data rate of $40 \mathrm{Mbps}$ ). After about 10 seconds, the QoS manager in process 1 first reacts to the delivered QoS by asking for more compute time and 
successfully adapts to the interference because the additional CPU capacity is available. Figure 6 shows the same scanario, but now process 2 uses a larger send-block size $(640 \mathrm{~KB})$. When the QoS manager in process 1 tries to adapt by requesting more compute time, it fails because sufficient CPU capacity is not available to accommodate the request.

The I/O interference visible in Figures 5 and 6 is due both to the overhead of TCP-ACK processing and to the fact that the streams scheduler fails to insulate the realtime ATM stream from the non-realtime TCP stream. In Solaris, processing an incoming packet involves preempting the current thread and running an internal kernel interrupt thread. The TCP input function is called in the interrupt context. TCP protocol processing occurs in the interrupt routine when an ACK is received. The increase in compute time of process 1 is an artifact of attributing the time used by interrupt and ACK processing for process 2 to process 1's account whenever it is interrupted.

One solution is to fix the accounting error to ensure that interrupt-processing time is not added to the compute time of interrupted threads. However, the interrupted realtime application can still miss its execution deadlines because its execution is delayed by the higher priority interrupt processing.

Instead of processing the incoming packet completely during an interrupt, we have introduced a new streams function called putschednext (shown in Figure 7) that postpones the protocol processing when an interrupt occurs. The IP streams module usually uses the putnext routine to pass data on to the TCP streams module. The new putschednext function, which is tied in with the RAP scheduler, replaces the putnext function in the TCP processing path. When invoked, it checks to see whether the interrupted thread is in the RAP class. If so, putschednext discontinues processing the packet and hands it to a background handler thread. Otherwise, it avoids the context switch and continues handling the packet itself.

We have observed that the streams scheduler uses a FCFS discipline. Before sending out the data for the ATM application, it processes buffered incoming data that was meant for processing by the streams background handler thread. It also first processes previously buffered outgoing data belonging to other I/O streams. The cost of processing such data is then charged to the ATM application. This FCFS nature results in processing TCP data in the context of the ATM application and causes interference with realtime data processing.

In AQUA, we have introduced a general purpose mechanism to solve the I/O interference problem and the problem of estimating CPU time needed for I/O. Our solution for the output (send) side extends the current Solaris implementation to make it sensitive to QoS needs of individual streams. An application indicates that a given stream is realtime by invoking a new ioct 1 option. A modified streams scheduler uses this information to shepherd the outgoing data through the protocol stack without any delay or buffering. It ensures that outgoing data for non-realtime streams is not processed first when processing data for a realtime stream. 
A: Compute time for

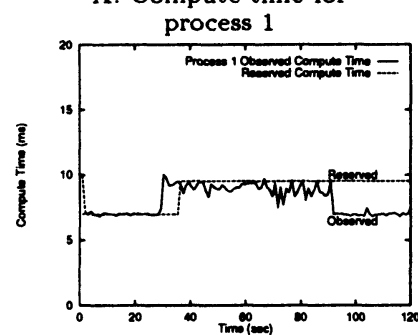

C: ATM send-data rate for process 1

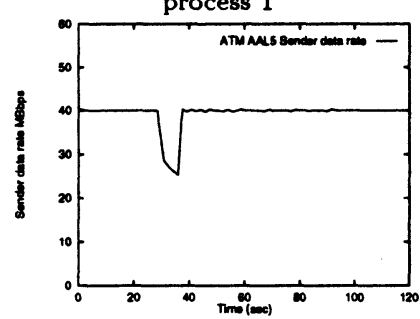

B: Rate of execution for process 1

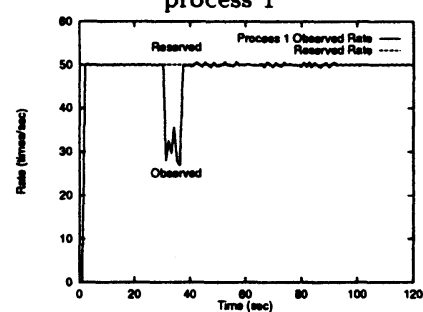

C: TCP throughput for process 2

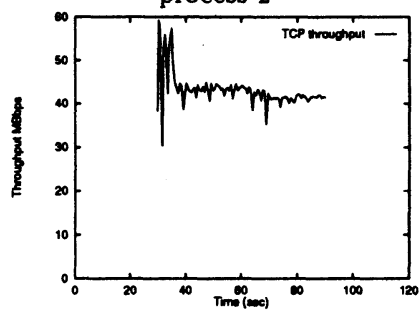

Figure 5 Successful QoS adaptation. Process 1 sends packets of size of $4 \mathrm{~KB}$ at a rate of $40 \mathrm{Mbps}$ at an execution rate of 50 times/sec, consuming $8 \mathrm{~ms}$ per period. Process 2 starts at time 30 , sends data with a TCP window size of $32 \mathrm{~KB}$ and a send-block size of $32 \mathrm{~KB}$.

A: Compute time for

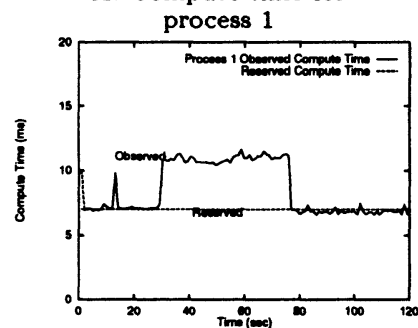

C: ATM send-data rate for process 1

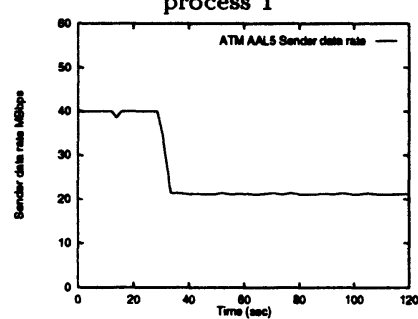

B: Rate of execution for

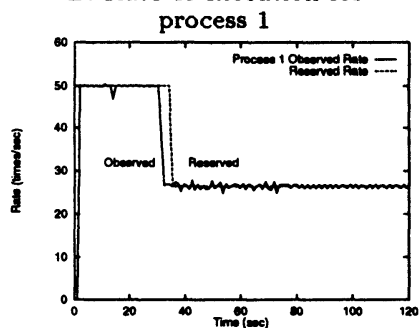

C: TCP throughput for process 2

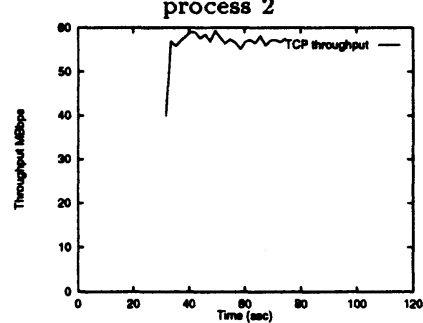

Figure 6 Unsuccessful QoS adaptation. The processes are the same as in Figure 5, but process 2 sends data in $640 \mathrm{~KB}$ blocks. 

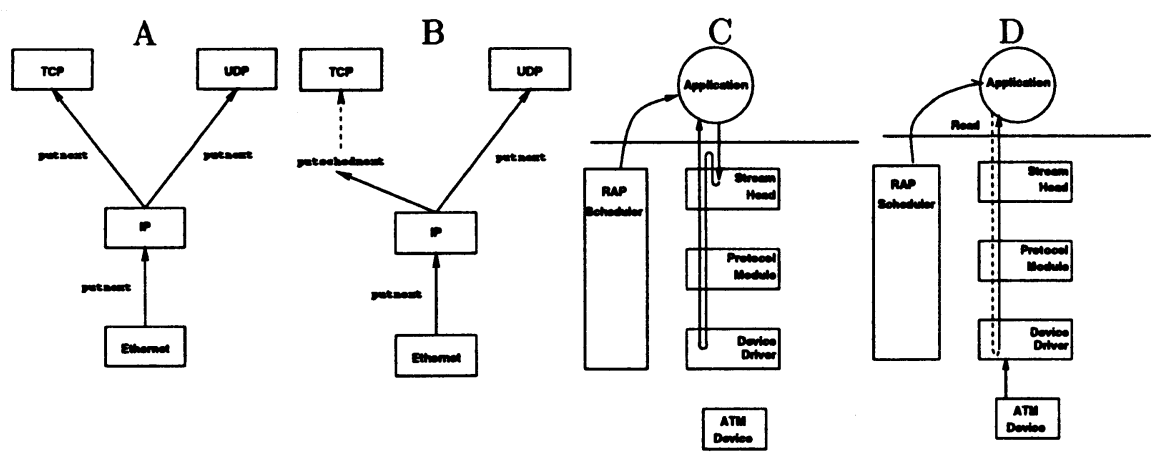

Figure 7 A (original) The IP streams module uses the putnext function to pass data on to either TCP or UDP streams modules. B (putschednext) To to provide better predictability, the IP streams module uses putschednext to pass data on to the TCP streams module C (AQUA sensitive sendpath) A new ioctl call allows creation of realtime streams; the modified streams scheduler only processes data belonging to a process that initiated the send. D (AQUA-sensitive receive path) Early demultiplexing by the device is necessary to ensure proper QoS. Incoming data is processed when the application issues a read call.

So far, we have only implemented the send side of this solution, but the receive side of protocol processing can also be made sensitive to QoS needs of realtime streams by generalizing the putschednext function. The networkinterface driver or the network card should be capable of determining the destination process for incoming data. An incoming packet is queued up in the lowermost module of a stream until the recipient process issues the read system call. This call allows RAP to perform protocol processing in the context of the recipient process and to charge the compute time appropriately. A similar solution for input part of protocol processing has been implemented in (Druschel \& Banga 1996) to improve protocol processing efficiency.

These modifications ensure that protocol processing costs are charged appropriately to the application that performs $\mathrm{I} / \mathrm{O}$ and that data is transferred according to the realtime nature of the application. The cost of interrupt processing itself can still interfere with an application and needs more study.

To demonstrate the effect of our modifications, we repeated the experiments involving an ATM-based application and the TCP-send application. Figure 8 shows the effect of using the AQUA sensitive send path and putschednext on the ATM send-data rate when an interfering TCP application is also present. The interference is significantly reduced compared to Figure 6 .

\section{RELATED WORK}

Govindan and Anderson (Ramesh Govindan 1992) proposed a new scheduling mechanism consisting of a split-level realtime scheduler based on the EDF (Earliest Deadline First) discipline. The authors were first to point out the 
A: Compute time for

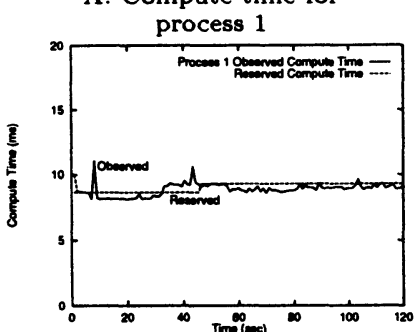

C: ATM send-data rate for process 1

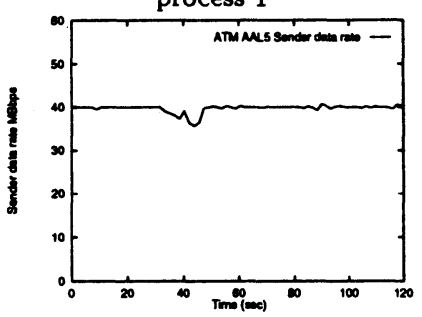

B: Rate of execution for process 1

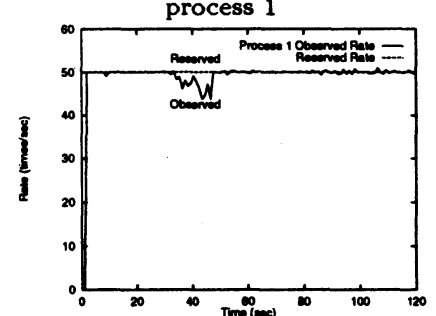

C: TCP throughput for process 2

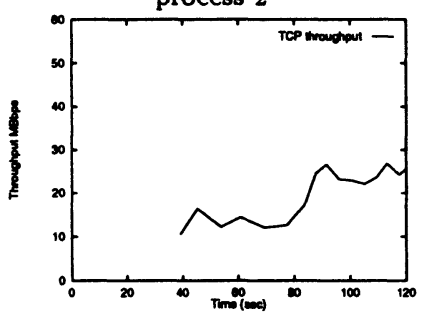

Figure 8 Same as in Figure 6, but process 1 declares its stream realtime via an ioctl call; the IP module uses the putschednext function for TCP data.

need for applications to accurately (and apriori) estimate their resource requirements. AQUA, in constrast, includes mechanisms to dynamically estimate the resource requirements of applications.

Under the Q-thread (Kawachiya \& Tokuda 1996) execution model, applications can specify a rate of execution, determine the amount of compute time available at that rate, and adapt to that compute time. The form of adaptation supported is similar to the AQUA's transparent adaptation model. Applications specify the adaptation policy to a QoS server, which initiates degradation.

OMEGA (Nahrstedt \& Smith 1995) supports end-to-end QoS management. QoS negotiation is performed by a QoS broker. The application-level QoS manager in AQUA is similar to the QoS broker; the AQUA negotiation library uses a protocol that is similar to the QoS-broker negotiation protocol.

An architecture for endsystem QoS, which is composed of components for specifying, mapping, and enforcing QoS has been developed in (Gopalakrishnan \& Parulkar 1994). An application specifies high-level QoS requirements based on predefined profiles. The framework maps these profiles into QoS specifications for all the resources used by the application. Applications make use of a realtime upcall to ensure CPU and network-I/O QoS.

Coulson et al. present the design of a QoS-controlled communications resource (Coulson, Campbell, Robin, Blair, Papathomous \& Shepard 1995). Their design provides an API for applications to specify QoS, proposes admission control tests for various classes of network traffic and explores methods to translate network-QoS requirements into $\mathrm{CPU}$ and memory requirements. 


\section{CONCLUSIONS}

We have demonstrated a way to provide predictable QoS for multimedia applications in an environment where applications do not know the exact resource requirements and both resource requirements and resource availability vary dynamically. AQUA presents a cooperative model of resource management.

To use the CPU resource, an application only specifies the required rate of execution, and an application-level CPU QoS manager dynamically estimates compute-time requirements. The CPU QoS manager reacts to resourcerequirement and availability changes to insulate applications from full QoS specification and negotiation.

We have explored integrated management of a composite resource like I/O, which consists of CPU, network-interface bandwidth, and bus bandwidth. The I/O-interference and inter-stream interference problems in Solaris streams highlight the problems inherent in existing I/O-resource implementation. Our extensions to the Solaris streams make the data paths sensitive to the QoS needs of applications. We ensure that data is transferred across the protocol stack taking into account realtime requirements while charging protocolprocessing costs to the corresponding application, and allowing a QoS manager to estimate the CPU needs for network-I/O.

\section{REFERENCES}

Coulson, G., Campbell, A., Robin, P., Blair, G., Papathomous, M. \& Shepard, D. (1995), 'The Design of a QoS-Controlled ATM-Based Communications System in Chorus', IEEE JSAC 13(4), 686-699.

Druschel, P. \& Banga, X. (1996), Lazy Receiver Processing, in 'Proceedings of the OSDI II', pp. 91-105.

Gopalakrishnan, R. \& Parulkar, G. M. (1994), Efficient Quality of service support in Multimedia Computer Operating systems, Technical Report WUCS-94-26, Washington University - Dept. of Computer Science.

Kawachiya, K. \& Tokuda, H. (1996), Q-thread: A new execution model for dynamic qos control of continuous-media processing, in '6th International NOSSDAV Workshop'.

Lakshman, K. (1997), AQUA: Adaptive Quality of Service Architecture, $\mathrm{PhD}$ thesis, Univeristy of Kentucky. http://www.dcs.uky.edu/ lakshman/thesis.

Nahrstedt, K. \& Smith, J. M. (1995), 'The QoS Broker', IEEE Multimedia 2(1), 53-67.

Ramesh Govindan (1992), Operating Systems Mechanisms For Continous Media, $\mathrm{PhD}$ thesis, UC Berkeley.

Yavatkar, R. \& Lakshman, K. (1995), A CPU Scheduling Algorithm for Continuous Media Applications, in 'Proceedings of the 5th International NOSSDAV Workshop, Durham, New Hampshire', pp. 223-226. 